Post-print version of:

Publisher: Wiley

Journal paper: International Journal for Numerical Methods in Biomedical Engineering, 33(8), e2839.

Title: Computational design and engineering of polymeric orthodontic aligners

Authors: S. Barone, A. Paoli, A.V. Razionale, R. Savignano

Creative Commons Attribution Non-Commercial No Derivatives License

(c) $\underset{B Y}{(9 C}=$

DOI Link: https://doi.org/10.1002/cnm.2839 


\title{
Computational design and engineering of polymeric orthodontic aligners
}

\author{
S. Barone ${ }^{1}$, A. Paoli ${ }^{1 \S}$, A. V. Razionale ${ }^{1}$, R. Savignano ${ }^{1}$ \\ ${ }^{1}$ University of Pisa, Department of Civil and Industrial Engineering \\ $\S$ Corresponding author
}

E-mail: s.barone@ing.unipi.it, a.paoli@ing.unipi.it, a.razionale@ing.unipi.it, roberto.savignano@for.unipi.it

\begin{abstract}
Transparent and removable aligners represent an effective solution to correct various orthodontic malocclusions through minimally invasive procedures. An aligner-based treatment requires patients to sequentially wear dentition-mating shells obtained by thermoforming polymeric disks on reference dental models. An aligner is shaped introducing a geometrical mismatch with respect to the actual tooth positions in order to induce a loading system, which moves the target teeth towards the correct positions. The common practice is based on selecting the aligner features (material, thickness, auxiliary elements) by only considering clinician's subjective assessments.

In this paper, a computational design and engineering methodology has been developed to reconstruct anatomical tissues, to model parametric aligner shapes, to simulate orthodontic movements and to enhance the aligner design. The proposed approach integrates computer-aided technologies, from tomographic imaging to optical scanning, from parametric modelling to finite element analyses, within a threedimensional digital framework.

The anatomical modelling provides anatomies, including teeth (roots and crowns), jaw-bones and periodontal ligaments, which are the references for the down streaming parametric aligner shaping. The biomechanical interactions between anatomical models and aligner geometries are virtually reproduced using a finite element analysis software. The methodology allows numerical simulations of patient-specific conditions and the comparative analyses of different aligner configurations.

In this paper, the digital framework has been used to study the influence of various auxiliary elements on the loading system delivered to a maxillary and a mandibular central incisor during an orthodontic tipping movement. Numerical simulations have showed a high dependency of the orthodontic tooth movement on the auxiliary element configuration, which should then be accurately selected in order to maximize the aligner's effectiveness.
\end{abstract}

\section{Keywords}

Orthodontic treatment, Polymeric aligner, Computational engineering, Computer Aided Design, Finite Element Analysis, Patient-specific modelling 


\section{Introduction}

Orthodontic appliances are designed to correct individual malocclusions by applying loads leading to specific tooth movements. Traditionally, the clinical practice makes use of fixed devices, which integrates metal archwires and brackets. In the last decades, the demand of minimally invasive alternatives has oriented the biomedical research toward the development of appliances, which combine the effectiveness in correcting tooth positions with the fulfilment of comfort and aesthetical issues [1]. Recently, transparent polymeric shells (aligners) having tooth-receiving cavities are becoming reliable solutions in the daily clinical practice. This orthodontic approach consists of incrementally moving teeth (target teeth) by wearing a plurality of successive aligners, where each aligner progressively repositions one or more of the patient's teeth by small amounts [1]. Pre-established geometrical mismatches (activation) between the aligner shape and the dental crown geometry generate three-dimensional force systems distributed all over the contact surfaces. A full treatment consists of a set of appliances with sequentially varying shapes, ranging from the initial anatomical geometry to the final tooth positions. A therapeutic treatment is planned on the basis of the diagnostic conditions and the patient's requirements by setting the aligner features, such as the mechanical properties of the polymeric material, the thickness of the shell, the amount of activation and the integration of addition devices (auxiliary elements) [2-4].

Transparent aligners were introduced in the 50s of the last century, though planning and manufacturing processes were initially based on specialized and manual procedures. These peculiarities restricted the use of aligners to light corrections requiring small sets of appliances. The development of Computer Aided Design (CAD) and Rapid Prototyping (RP) techniques has allowed an industrial approach for both planning orthodontic treatments and manufacturing polymeric aligners $[5,6]$.

A CAD-based procedure is based on the digitalization of several steps of the overall process: from the reconstruction of patients' anatomies to the manufacturing of reference models. Digital reconstructions of dental crowns are carried out by optically scanning either a plaster model or the patient's mouth [7]. Segmentation tools are used to extract individual tooth shapes from the 3D scanned model. Specialized technicians plan single tooth movements by dedicated CAD software [6]. The CAD planning allows geometrical and functional constraints to be preliminarily verified, thus guiding the technician during the tooth re-positioning process. The orthodontic treatment is partitioned into smaller sequential movements of the teeth: from the initial actual condition to the final expected outcome [6]. RP methodologies are used to manufacture physical dentition moulds of each sequential step. The customised aligners are produced from the prototyped moulds (reference models) by a vacuum thermoforming process. A polymeric sheet is shaped over each prototyped mould and trimmed to obtain the final configuration.

The CAD-based procedures are fast and repeatable leading to a better management of the overall processes [6]. However, planning an accurate orthodontic treatment is still a challenge since tooth movement prediction is always based on the clinical expertise. The use of polymeric aligners introduces uncertainties regarding location, distribution and intensity of the loading system, which cannot be solved by the current CAD-based practice. The loading system depends on the polymeric material properties, the amount of activation 
programmed into the aligner and the complete tooth anatomies, which include crowns and roots [8]. Moreover, aligner-based treatments could require the use of auxiliary elements, such as attachments and geometrical singularities (divots), to assure specific orthodontic movements. In this context, the knowledge of how transparent aligners deliver forces and moments to tooth surfaces would allow the design of optimized appliances leading to more efficient orthodontic treatments [9].

Some attempts to evaluate loads delivered by aligners to dentitions have been made by experimental tests. An in-vivo study was carried out in [10] to measure buccal tipping movements of the upper first premolar through a pressure-sensitive film approach. A film was interposed between dentition and aligner during the treatment in order to obtain measurements of the force imparted by the appliance. Other approaches were based on the use of multi-axis force/torque transducers on replicated dental arches made of polymeric materials $[2,3,11-13]$. These measurements were characterized by a certain degree of approximation since the dentoalveolar anatomy was considered as made of homogenous structure and the model was made of a single material, which had different mechanical properties with respect to the biological tissues. Few models were developed by taking into account multi-material layouts [14]. Moreover, these in-vitro studies were limited on measuring forces on a single tooth (maxillary central incisor) and the analysis of further scenarios would have required manufacturing different aligners and tooth resin models.

In the orthodontic field, a numerical simulation could provide quantitative and detailed data on the biomechanical response occurring during treatments [15]. In particular, the Finite Element Method (FEM) represents a flexible cost- and time-saving solution to analyse orthodontic features and optimize their design. Finite Element Analyses (FEA) have been used to asses stress distributions in periodontal ligaments (PDL) and alveolar bones [16, 17], to predict tooth displacements [18], to optimise the design of brackets and archwires [19]. However, in scientific literature very few attempts have been made to study tooth-aligner interactions by finite element models [20-22] and no documentation exists on the study of orthodontic auxiliary elements by exploiting numerical analyses.

This paper introduces a Computational Design and Engineering (CDE) framework, which allows patientspecific simulations of mechanical interactions between dental tissues and polymeric aligners. The framework is articulated in the following modules: 1) reconstruction of anatomical tissues, 2) parametric modelling of aligner shapes and 3) finite element modelling of tooth-aligner interactions.

Complete anatomical data, including crown, root and bone tissues, are reconstructed by integrating tomographic and optical scanning methodologies. Parametric geometries are modelled to design aligner configurations on the basis of anatomical and functional requirements. Anatomical reconstructions and aligner geometries are then integrated within a finite element modeller. The numerical analyses provide the information about the biomechanical loading systems in different orthodontic scenarios. The computational approach allows a knowledge-based design of the most appropriate aligner features taking into account individual demands. 
In this paper, the CDE framework has been exploited to study the effects of various auxiliary elements on the loading system delivered to a maxillary and a mandibular central incisor simulating an orthodontic tipping movement.

\section{Fundamentals of polymeric aligners}

\subsection{Force system, Centre of Resistance and Centre of Rotation}

An orthodontic movement is a mechanobiological process provided by a loading system, which is represented by a combination of forces and moments $(\mathbf{F}, \mathbf{M})$ applied to the crown of a dental structure [23]. A specific tooth movement is determined by the equivalent force system $\left(\mathbf{M}_{\mathbf{e}}, \mathbf{F}_{\mathbf{e}}\right)$, which is referenced to the Centre of Resistance $\left(\mathrm{C}_{\mathrm{RES}}\right)$ [24]. The concept of $\mathrm{C}_{\mathrm{RES}}$ of a tooth is analogous to the concept of centre of mass except that it is not related to a free body. Indeed, a tooth is not free to move in response to a force since it is constrained in the alveolar complex by the periodontal structure and, consequently, a point analogous to the centre of mass is introduced as $\mathrm{C}_{\mathrm{RES}}$ [25]. The position of the $\mathrm{C}_{\mathrm{RES}}$ depends on the anatomical data and, in particular, on the root length. Identical forces applied to teeth having different root lengths may produce different effects, since the movement resulting from a force delivered at the tooth crown depends on the distance of the force's line of action from the $\mathrm{C}_{\mathrm{RES}}[26]$.

The type of tooth movement depends on the location of the centre of rotation $\left(\mathrm{C}_{\mathrm{ROT}}\right)$ defined as the point around which the actual tooth movement occurs. In particular, the $\mathrm{C}_{\mathrm{ROT}}$ varies with the moment-to-force ratios $\left(\mathrm{M}_{\mathrm{e}}: \mathrm{F}_{\mathrm{e}}\right)$ [24]. The ratio $\mathrm{M}_{\mathrm{e}}: \mathrm{F}_{\mathrm{e}}$ provides a description about the type of orthodontic movement, whereas the individual $\mathrm{M}_{\mathrm{e}}$ or $\mathrm{F}_{\mathrm{e}}$ absolute values are related to the amount of tooth movement [27]. The orthodontist acts on the appliance design in order to get the appropriate $\mathrm{M}: \mathrm{F}$ and, consequently, the equivalent $\mathrm{M}_{\mathrm{e}}: \mathrm{F}_{\mathrm{e}}$ value, which gives the required $\mathrm{C}_{\mathrm{ROT}}$ and a specific tooth movement.

\section{$2.2 \quad$ Typical tooth movements}

A tooth can be subjected to two types of movements: translation and rotation. In clinical practice, these movements can be combined into the following four main categories [28].

- Uncontrolled tipping - movement originating when the $C_{R O T}$ approaches the $C_{R E S}$ (Figure 1-a).

This movement occurs when a single force is applied to the tooth crown. The equivalent force system at the $\mathrm{C}_{\mathrm{RES}}$ is composed by a force and a moment. The tooth rotates around the $\mathrm{C}_{\mathrm{RES}}$ while translating. This movement is defined as "uncontrolled tipping" if the rotation occurs around an axis different from the tooth long axis, otherwise it is generally defined as rotation. When the $\mathrm{C}_{\mathrm{ROT}}$ overlaps the $\mathrm{C}_{\mathrm{RES}}$, the equivalent force system at the $\mathrm{C}_{\mathrm{RES}}$ is composed by a single moment, which induces the tooth to a pure rotation around the $\mathrm{C}_{\mathrm{RES}}$.

- Translation - movement originating when the $C_{R O T}$ is located at an infinite distance from the $C_{R E S}$ (Figure 1-b). 
This movement occurs when a force-moment system is applied to the crown surface in order to obtain an equivalent force system at the $\mathrm{C}_{\mathrm{RES}}$ composed by a single force. In this case, the tooth translates accordingly to the force direction.

- Controlled tipping - movement originating when the $C_{R O T}$ is moved down to the root apex (Figure $1-c)$.

This movement occurs when an anti-tipping moment is added at the crown level. The equivalent force system at the $\mathrm{C}_{\mathrm{RES}}$ is composed by a force and a moment and the tooth rotates around the root apex.

- Root movement - movement originating when the $C_{R O T}$ is moved at the crown level (Figure 1-d).

In this case, the force-moment system generates a tooth rotation around the crown tip.
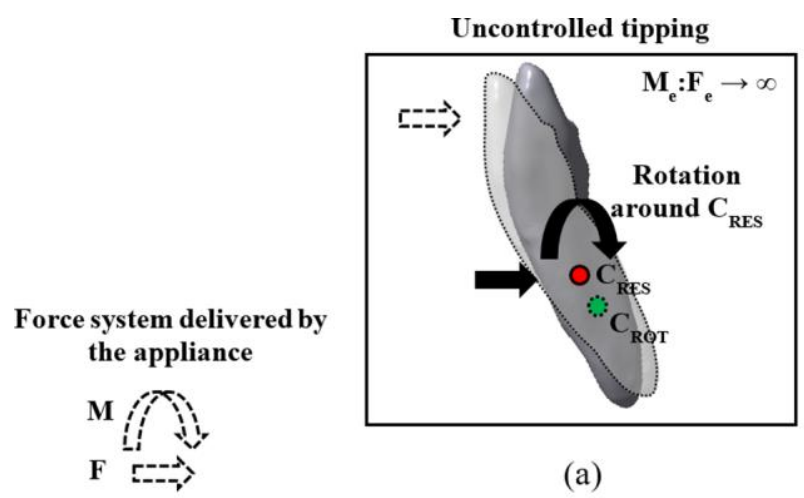

(a)

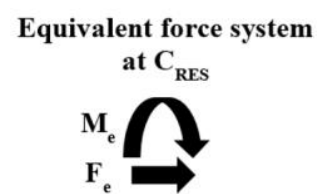

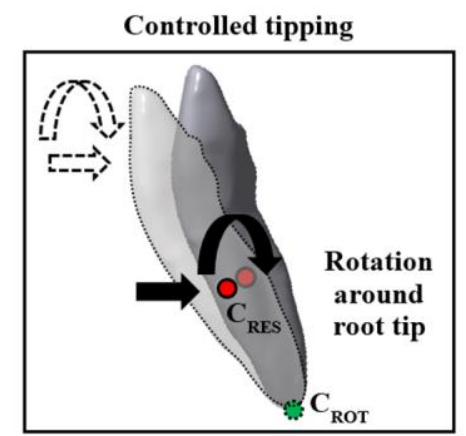

(c)

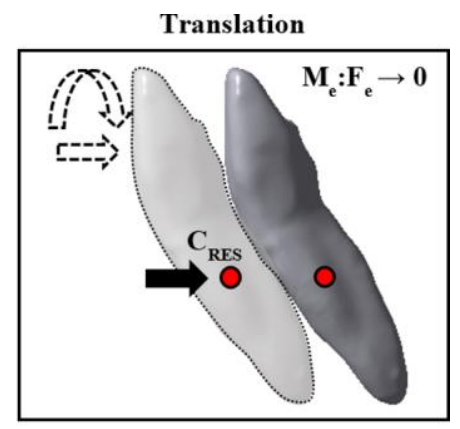

(b)

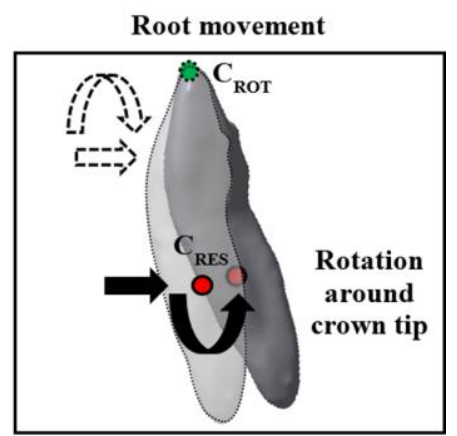

(d)

Figure 1 - Orthodontic tooth movements: uncontrolled tipping (a), translation (b), controlled tipping (c), root movement (d).

\subsection{Creation of a loading system}

The load delivering modalities depends on the specific configuration of the orthodontic appliance. For instance, a fixed appliance has a highly defined contact area, which is represented by the bracket. In this case, the load is usually supposed to be transferred at the centre of the bracket and the M:F value can be defined with respect to the bracket itself. If removable polymeric aligners are used, the force system is generated by the geometrical mismatch between tooth and appliance, and transferred to a wider and less defined contact area. Moreover, auxiliary elements, as attachments or divots (Figure 2), can be used to 
facilitate the load transfer between the appliance and the dentition through the modification of their contact interface.

Attachments are made of dental composite material, which is polymerized on the crown surface (Figure 2-a). Divots can be obtained as punctual introflexions of the aligner (Figure 2-b) by using pliers with spherical extremities. Auxiliary elements represent unavoidable features in order to obtain some critical orthodontic movements through a better definition of the contact area, a local increase of the mismatch in specific points and an accurate load control in terms of intensities and directions.

In common practice, the use of auxiliary elements is demanded to the clinician's expertise without any certified protocol based on scientific evidences. However, an accurate analysis of auxiliary element influence on the dental movement is of utmost importance. In particular, disposition, shape and number of attachments and/or divots would require an optimization analysis to increase the treatment predictability and effectiveness.

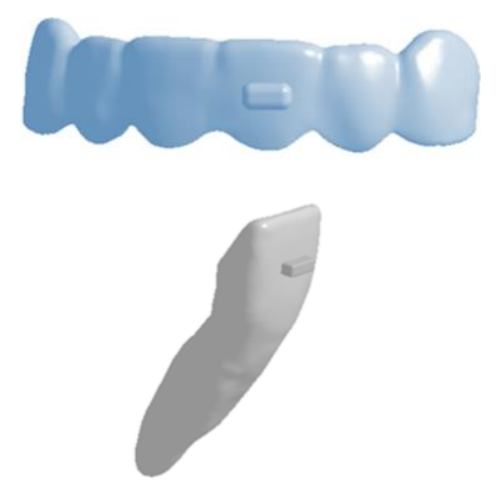

(a)

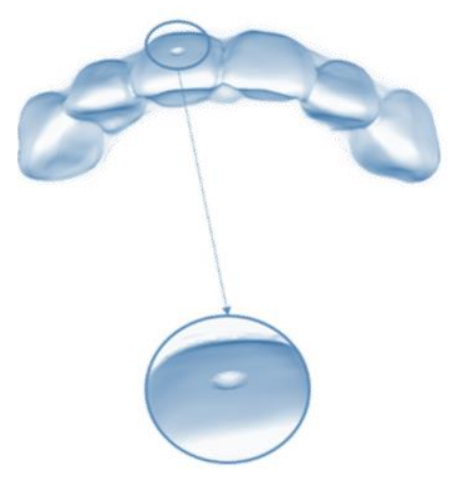

(b)

Figure 2 - Example of auxiliary elements used to enhance orthodontic movements: attachment (a) and divot (b).

\section{$3 \quad$ Materials and methods}

In this paper, a $\mathrm{CDE}$ framework has been developed with the aim at simulating patient-specific tooth-aligner interactions. The approach provides an accurate prediction of the biomechanical parameters $\left(\mathrm{C}_{\mathrm{RES}}\right.$ and $\left.\mathrm{M}_{\mathrm{e}}: \mathrm{F}_{\mathrm{e}}\right)$ by modelling complete dental anatomies and controlling aligner activations. The framework is articulated in the following modules: 3D reconstruction of dental tissues including crown and root tissues, modelling of aligner parametric geometries, modelling of tooth-aligner interactions.

\subsection{Reconstruction of complete anatomical models}

Patient's anatomical models, composed of alveolar bones, teeth and periodontal ligaments, are obtained by combining digital data extracted from Cone Beam Computed Tomography (CBCT) and optical scanning. In this work, an optical scanner based on a coded structured light approach is used to acquire plaster models and to provide accurate reconstructions of all the visible tissues: from crowns to gingiva. The overall tissues are segmented into disconnected regions, representing distinct crown geometries and gingiva surfaces, through a semi-automated procedure based on a curvature detection algorithm [29]. 
A CBCT imaging technique is used to reconstruct jawbones and individual teeth including crown and root anatomies. A CBCT scan yields a stack of slices corresponding to cross-sections of a maxillofacial volumetric region. CBCT data are stored in a sequence of Digital Imaging and Communications in Medicine (DICOM) images. An imaging slice is a 2D matrix of grey intensity values representing the $\mathrm{x}$-ray attenuation of different anatomical tissues.

Three-dimensional models of jawbones are obtained by segmenting the volumetric CBCT data with a specific grey intensity value (isovalue). The reconstruction of distinct tooth anatomies is not so straightforward. Most of the existing segmentation techniques are based on contouring each tooth through time-consuming slice-by-slice procedures. In this paper, DICOM images are segmented by adopting the methodology introduced in [30]. This method is based on processing four multi-planar reformation images, which are obtained, for each tooth, on the basis of anatomy-driven considerations. The reformation images greatly enhance the clearness of the target tooth contours, which are then extracted and used to model the overall 3D tooth shape through a B-spline representation. Four reference planar sections are automatically extracted as passing from the tooth axis and oriented along the buccolingual direction, the mesiodistal direction and the two directions disposed at $45^{\circ}$ with respect to these meaningful clinical views. These reference sections are used to outline the tooth by interactively tracing four different $2 \mathrm{D}$ tooth contours $\left(C_{i}\right)$ as shown in Figure 3-a. The four contours are used to automatically extract a set of B-spline curves. Each slice perpendicular to the tooth axis (transverse slice) intersects the $C_{i}$ contours in eight points that are used as control points to compute a parametric B-spline curve of degree 2 (Figure 3-b). For each slice, 100 points are evaluated on the B-spline curve in order to obtain a point cloud representing the overall tooth shape.

The greatest benefit of this methodology consists in providing reliable approximations of individual tooth roots, by interactively contouring a few significant images created from the whole CBCT data set. The processing time is greatly reduced with respect to standard cumbersome slice-by-slice methods usually proposed through medical imaging software. However, CBCT data are not always accurate enough to reconstruct crown surfaces, which are used as mating references to model aligner shapes. In this paper, the multi-source data obtained by using optical and tomographic scanning are merged to create accurate multibody dental models. The segmented crown geometries obtained by optical scanning and CBCT imaging are coarsely aligned into a common reference frame by manually selecting at least three common points. A refinement of the initial alignment is then performed by a fine registration procedure based on the Iterative Closest Point (ICP) technique. The crown geometries obtained by processing DICOM images are removed by means of a disk vertex selection algorithm. Each point of the optical crown mesh is projected into a point on the CBCT mesh. This point is the centre of a sphere, which is used to select the CBCT crown points to be removed. Complete tooth models are obtained by a Poisson surface reconstruction approach [31]. This procedure provides dental models including the most accurate representations of both roots and crowns.

The tooth axes are defined accordingly to [32] and centred at the $C_{\text {RES. }}$. The $z$-axis is defined approximately perpendicular to the occlusal plane and associated with the lower moment of inertia of its geometrical model. The mesiodistal ( $y$-axis) and the buccolingual ( $x$-axis) directions are taken orthogonal to the $z$-axis and 
determined by analysing the principal inertia components of the planar section obtained by slicing the tooth crown with a plane perpendicular to the $z$-axis, $3 \mathrm{~mm}$ far from the crown tip.

Tooth models are improved by modelling Periodontal Ligament (PDL) geometries. PDL tissues cannot be easily visualized and reconstructed using CBCT since the slice thickness is similar or even greater than the ligament space (about $0.2 \mathrm{~mm}$ ) [33]. For this reason, the PDL is modelled by detecting the interface area between bone and tooth models. In practice, a film (thickness $=0.2 \mathrm{~mm}$ ) is created from the external surface of the tooth (Figure 4-a). The film and the bone are intersected in order to obtain the PDL geometry (Figure 4-b). The external surface of the film is used for a Boolean subtraction from the jawbone in order to create the PDL housing (Figure 4-c). Figure 4-d shows a section of the final orthodontic model obtained for a central tooth.

Figure 5 schematizes all the steps concurring in the creation of the final patient's orthodontic model composed of distinct teeth, periodontal ligaments and bone tissue. The tooth models can be individually rotated and/or translated within a three-dimensional virtual environment on the basis of the specific therapeutic requirements.

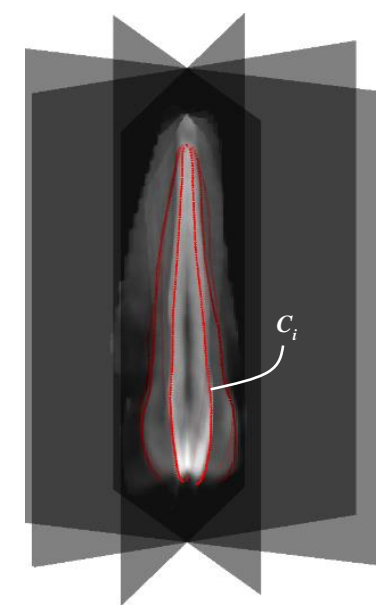

(a)

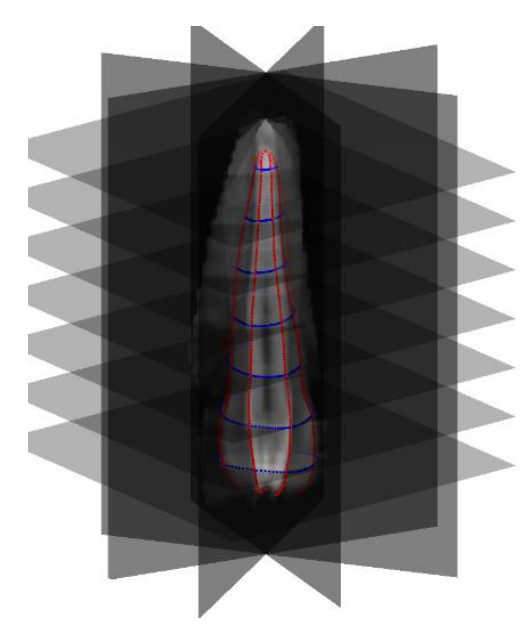

(b)

Figure 3 - Four reference planar sections along with the 2D tooth contours for a superior canine tooth (a), B-spline curves computed for the transverse slices (b).

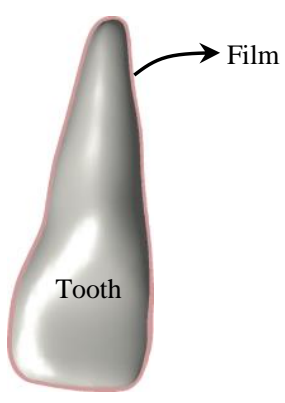

(a)

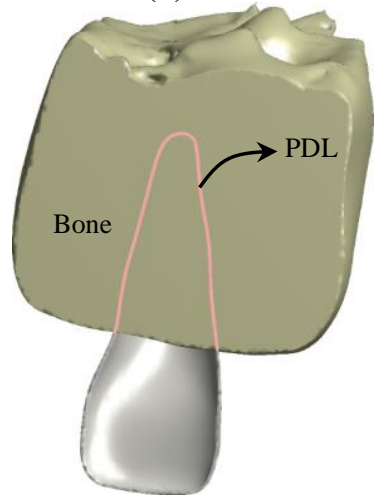

(b)

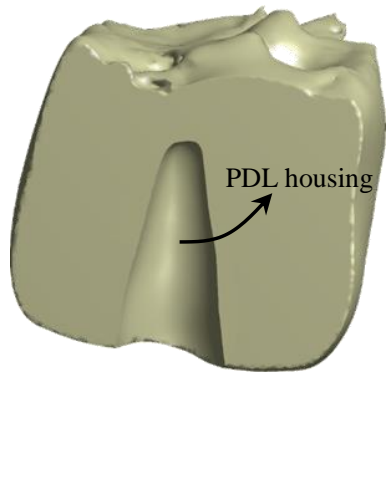

(c)

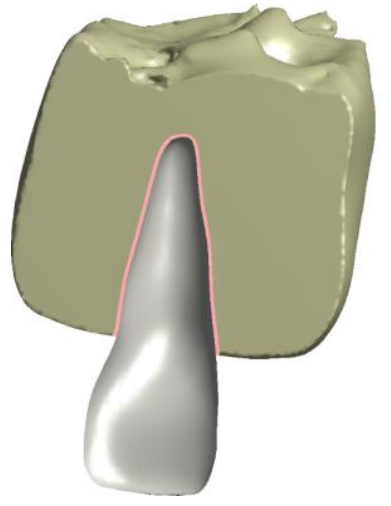

(d)

Figure 4 - Film creation from the external surface of the tooth (a), PDL geometry reconstruction by intersecting bone and film (b), PDL housing definition by subtracting the external film surface from the bone (c), orthodontic model of a single tooth (d). All the figures have been created by sectioning the anatomical models in order to highlight the involved structures. 


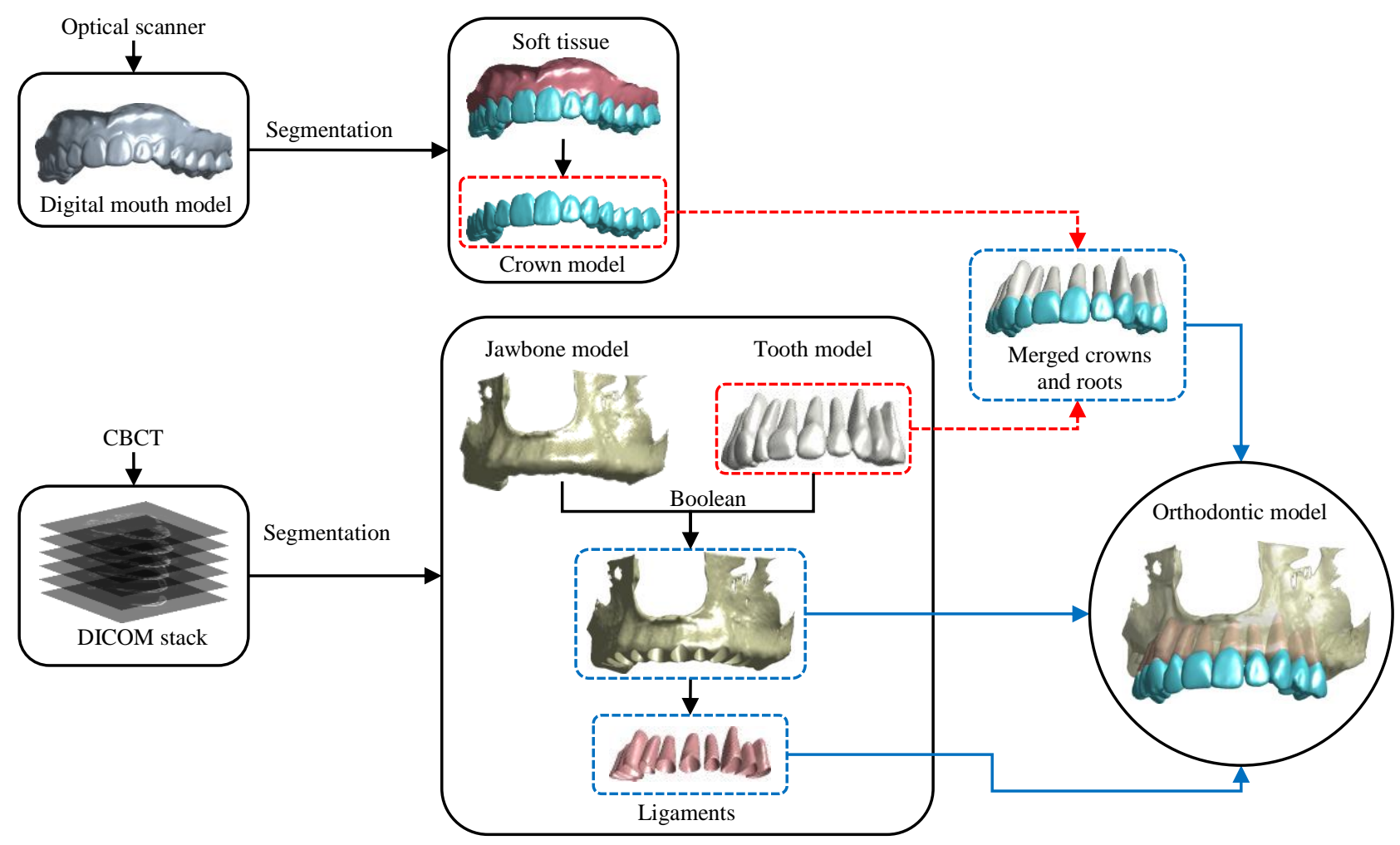

Figure 5 - Scheme summarizing the creation of the final patient orthodontic model.

\subsection{The aligner modelling process}

Digital aligner shapes are obtained by mating dental crown surfaces and taking into account thermoforming requirements. Individual crowns are merged and undercut regions are manually removed in order to define a unique, thermo-formable layer. The layer is thickened to create a uniform shell of $t=0.7 \mathrm{~mm}$, which originates from the mean thickness of the thermoplastic material disk $(0.75 \mathrm{~mm}$ thick) before the thermoforming process [34]. This procedure guarantees an optimal fit between the mating surfaces of tooth crowns and appliance [22].

Attachments and divots are created through Boolean operations between tooth crown, aligner and primitive parametric auxiliary shapes, as shown in Figures 6. The prismatic geometry of an attachment is created on the tooth crown surface sizing the three principal dimensions along the $x, y, z$ tooth axes and setting the fillets on all the edges. The geometry of a divot is created from a sphere sizing the radius $r$. The parametric modelling of auxiliary elements can be summarized as follows:

Attachment (Figure 6-a)

- creation of a prismatic shape on the target tooth crown surface (B);

- creation of a $t=0.7 \mathrm{~mm}$ thick body from the prismatic shape (C);

- Boolean subtraction between the two prismatic shapes $(\mathrm{C}-\mathrm{B}=\mathrm{E})$;

- Boolean subtraction between the aligner and the thick body prismatic shape $(\mathrm{A}-\mathrm{C}=\mathrm{D})$;

- Boolean addition between the cropped aligner and the attachment shell $(\mathrm{D}+\mathrm{E})$;

Divot (Figure 6-b) 
- creation of two concentric spheres with radii $r(\mathrm{~B})$ and $r-t(\mathrm{C})$, and the centres located on the external surface of the aligner;

- sectioning of the two spherical shapes with the aligner external surface (B sectioned by $A=B^{\prime}, C$ sectioned by $\left.\mathrm{A}=\mathrm{C}^{\prime}\right)$;

- Boolean subtraction between the aligner and the bigger sphere $(\mathrm{A}-\mathrm{B}=\mathrm{D})$;

- Boolean subtraction between the two sliced spheres $\left(\mathrm{B}^{\prime}-\mathrm{C}^{\prime}=\mathrm{E}\right)$;

- Boolean addition between the cropped aligner and the divot shell (D+E).

The parametric modelling process allows the study of loading systems in different geometrical configurations obtained by modifying the aligner attributes. In particular, amount and distribution of the aligner thickness, layout and dimensions of auxiliary elements can be adjusted to obtain the expected orthodontic responses on the basis of the numerical simulations.

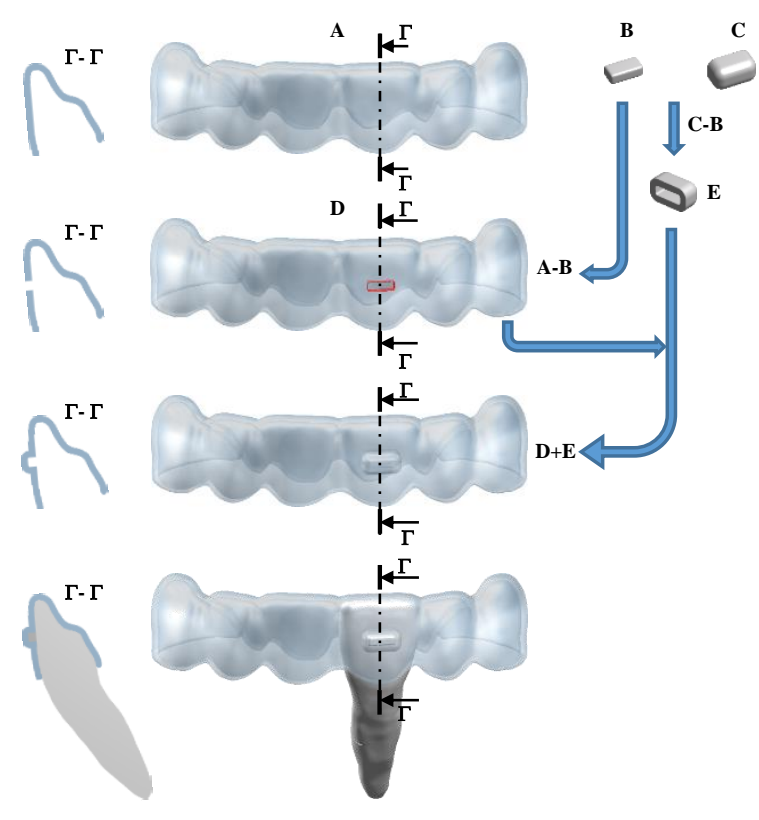

(a)

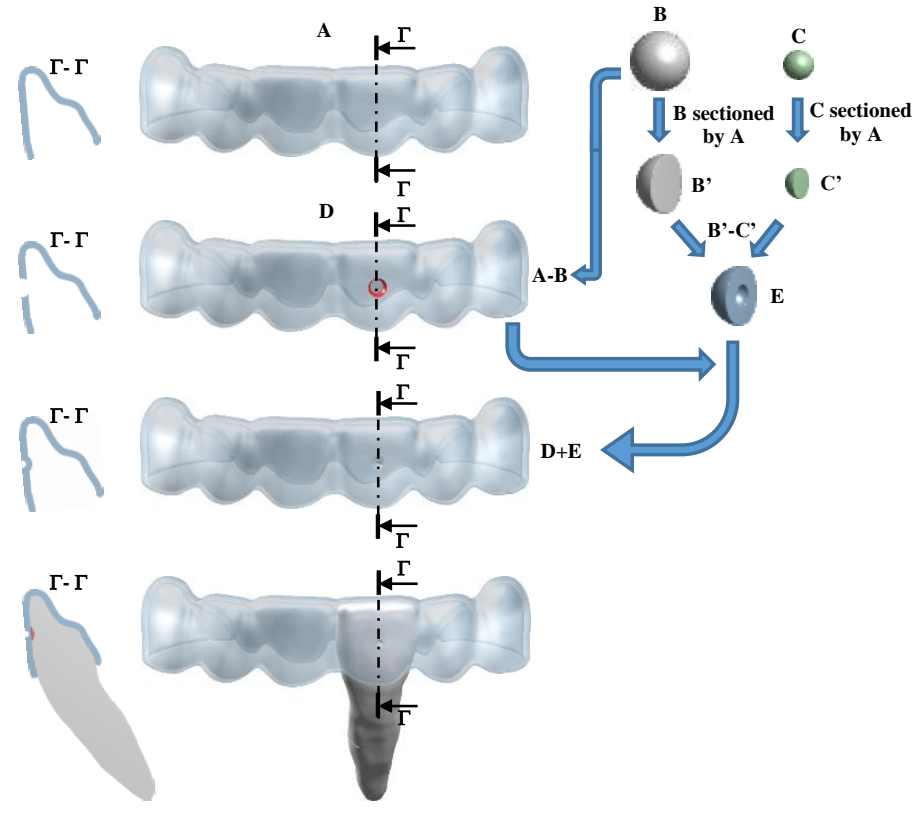

(b)

Figure 6 - Attachment (a) and divot (b) parametric modelling.

\subsection{Modelling of tooth-aligner interactions}

The mechanical responses of bones, teeth, attachments and aligners are described by a linear elastic constitutive model (Table 1). The elasticity parameters of bone, tooth and attachment are much greater with respect to the PDL parameters (in a range of four to five orders of magnitude) [35]. For this reason, the linear elastic model can be considered adequate. This simplification could not be considered realistic for the aligner material. However, in the present work, only the initial tooth movement is considered and for this circumstance also the behaviour of the aligner can be considered linear elastic [34, 36]. Moreover, teeth and bones are supposed to be made of homogenous and isotropic materials, without discriminating internal tissues (enamel, pulp, dentin for teeth, and cortical, cancellous for bones). This assumption appears to be reasonable due to the higher stiffness of tooth and bone with respect to the PDL. Consequently, any change 
in the elasticity parameters due, for example, to a variation in the cancellous bone density or to anisotropic or non-homogenous mechanical behaviour do not have a significant influence on the initial orthodontic tooth movement [35].

The investigation of the ligament in-vivo behaviour is not a trivial task due to its small size (about $0.2 \mathrm{~mm}$ thickness). For this reason, most of the scientific literature has investigated the mechanical properties of the PDL through experimental analyses, thus developing five different models: linear elastic, bilinear elastic, viscoelastic, hyperelastic and multiphase [37]. The linear elastic model has demonstrated that is able to approximate the PDL behaviour for the initial phase of the orthodontic movement if the PDL maximum strain value is lower than $7.5 \%$ [38]. In this paper, the volumetric finite strain viscoelastic model is implemented as proposed in [39], since the simulated orthodontic movements do not fulfil the maximum strain requirements.

The auxiliary attachments are made of dental composite material and modelled of the same tooth material properties. The aligners are obtained from a polyethylene terephthalate glycol-modified (PETG) thermoplastic disc. The mechanical properties have been evaluated through a set of tensile tests carried out under different experimental conditions to take into account the thermoforming process and the specific operative environment [36]. In particular, the specimens have been manufactured by a thermoforming procedure and subjected to an aging treatment in a solution reproducing the biochemical behaviour of human saliva.

A crucial problem to be faced is related to the loading conditions due to the interactions between aligners and crown geometries. In particular, the loading conditions are activated by creating proper mismatches between the target crowns and the aligner. A geometrical mismatch is obtained by translating/rotating a tooth on the basis of the target orthodontic movement.

A numerical simulation requires the creation of finite element models, which integrate the structures anatomical tissues - polymeric aligner.

Teeth and their respective PDLs are joined by a bonded contact. Corresponding nodes cannot separate each other and a perfect adhesion between contact surfaces, without mutual sliding or separation, can be assumed. The same constraint is used to join bone and ligaments, while the bone nodes not in contact with the periodontal ligaments are fixed in all directions.

A finite element modeller (Ansys ${ }^{\circledR} 14$ ) resolves the activation mismatch between crowns and aligner by determining the equilibrium between the bodies. Contact interfaces between teeth and aligner are set as frictionless, with maximum residual penetration of $0.01 \mathrm{~mm}$, which provides the best accuracycomputational time ratio. This can be considered a reasonable choice due to the existent dissimilarity between the aligner polymeric material and the dentition biological tissue, taking also into account the presence of saliva. An augmented Lagrangian formulation is used to simulate the contact. The non-linear problem is solved by using the Newton-Raphson residuals method based on force and moment convergence values. The number of initial sub-steps for each simulation is set as 150 , whereas the contact stiffness is automatically updated at each iteration. During the Newton-Raphson iterations, the mismatch is checked 
with respect to the maximum allowable penetration tolerance value. An undesired initial mismatch between the aligner geometry and non-target teeth could occur due to the meshing process. For this reason, the "adjust to touch" contact option is used for those contact pairs in order to remove all the undesired initial gaps or penetrations.

\section{$4 \quad$ A case study}

\subsection{Configuration setting}

The CDE framework has been used to analyse the effects of different auxiliary elements on an uncontrolled tipping movement of maxillary and mandibular central incisors. The reference anatomical models include six frontal teeth for both mandibular and maxillary tissues. The study has been conducted simulating aligners with different auxiliary elements located on the incisor teeth. In particular, the following configurations have been analysed (Figure 7): standard (without auxiliary elements), single divot, double divots, vertical and horizontal attachments. Vertical and horizontal attachment geometries have been created with sizes $1 \times 1.5 \times 3$ $\mathrm{mm}$ and $1 \times 3 \times 1.5 \mathrm{~mm}$ on the $x, y$ and $z$ directions, respectively. These values have been derived from the clinical practice. The divot geometry has been created with $r=1 \mathrm{~mm}$ in order to guarantee a penetration of $0.3 \mathrm{~mm}$ between the aligner and the target tooth crown. The numerical simulations have been carried out changing the locations of the auxiliary elements over the crowns of the target teeth. In particular, the attachments and the divots have been vertically translated along the $z$-axis by varying the height $(h)$ between $-1 \mathrm{~mm}$ and $1 \mathrm{~mm}$ with reference to the centre of the crown surface (Figure 8-a). Moreover, the divot pairs have been moved along the $y$-axis in order to modify their mutual distance $(b)$ between $2.5 \mathrm{~mm}$ and $3.5 \mathrm{~mm}$ (Figure 8-b).

The finite element models have been created by using quadratic 10-node tetrahedral elements (SOLID187). These elements are suitable to model irregular shapes as those characterizing the reconstructed patientspecific anatomical models. The meshes have approximately included 132000 nodes and 83000 elements for the maxillary segment and 128000 nodes and 80000 elements for the mandibular segment. The model sizes have slightly varied between the different scenarios due to the various auxiliary configurations.

According to [25], three simulations have been run in order to determine the $\mathrm{C}_{\mathrm{RES}}$ by sequentially applying a moment of $1.5 \mathrm{~N} \mathrm{~mm}$ parallel to the coordinate system axes of each target tooth.

The uncontrolled tipping movements have been modelled by activating a loading condition through a rotation of each target tooth around the $\mathrm{C}_{\mathrm{RES}}$, along $y$-axis by $-0.3^{\circ}$ (Figure $10-\mathrm{a}$ ). The initial mismatch has ranged from $0.09 \mathrm{~mm}$ to $0.36 \mathrm{~mm}$. The initial penetrations generated by the divots have resulted higher than those occurring by using the attachments (Figure 10-b). 


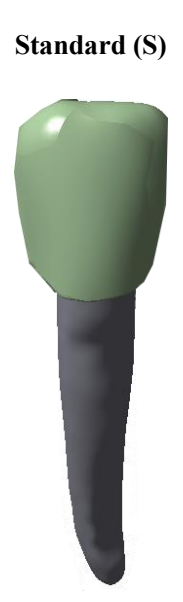

(a)

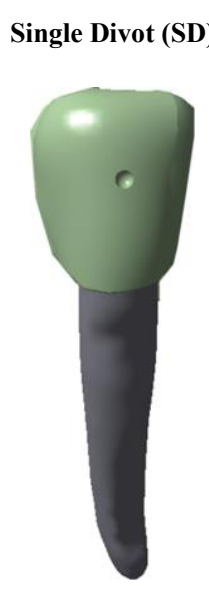

(b)

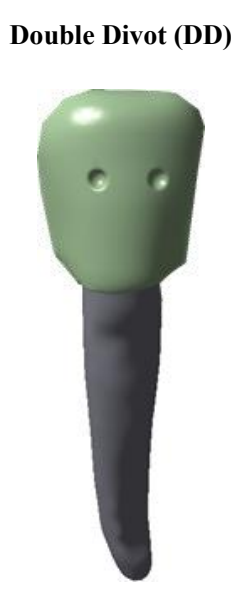

(c)

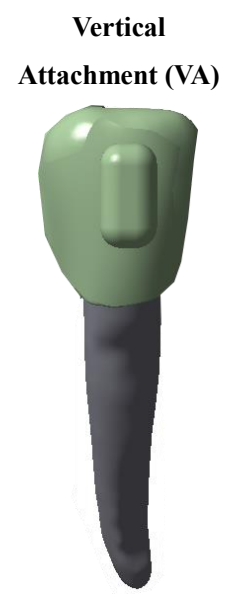

(d)
Horizontal

Attachment (HA)

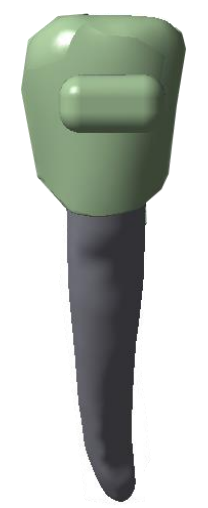

(e)

Figure 7 - Different geometrical configurations of the aligner design in correspondence of a target tooth.

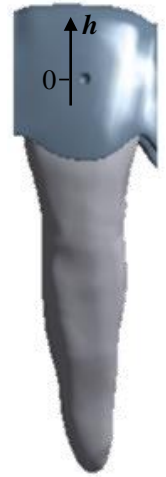

(a)

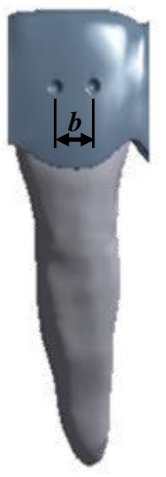

(c)

Figure 8 - Parameters used to modify the auxiliary elements position: distance from the crown centre (a) and mutual distance between divots for the double divot configuration (b).

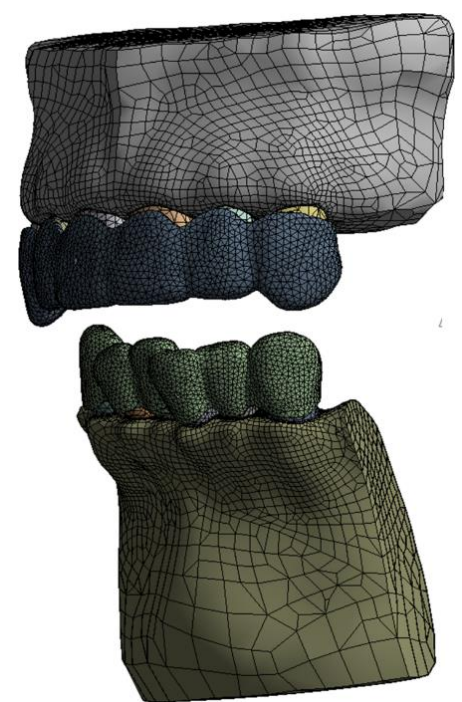

Figure 9 - Example of a meshed model used for the numerical simulations. 


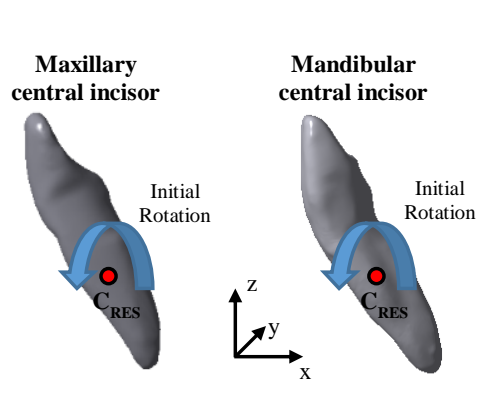

(a)

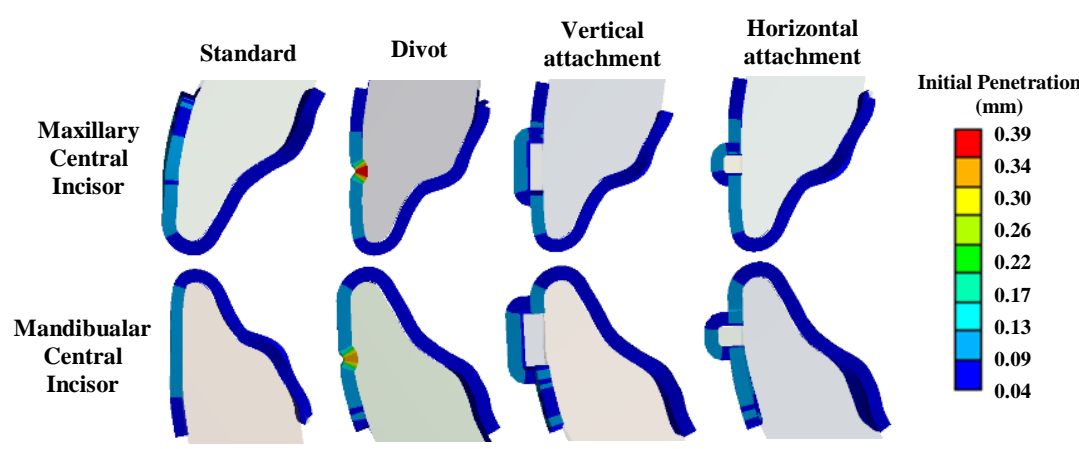

(b)

Figure 10 - $\mathrm{C}_{\mathrm{RES}}$ and tooth rotation (a) to create the initial penetration (b) introducing the loading conditions.

\subsection{Numerical results}

The computational time for each simulation was approximately between 1.5 and 2.5 hours, using a Workstation based on Intel Xeon CPU E3-1245 v3@3.40GHz and 16 GB of RAM. The numerical results have been expressed in terms of the force system delivered by the aligner to each incisor tooth.

As described in Section 2, the most significant parameters to assess the orthodontic movements are the ratio $\mathrm{M}: \mathrm{F}$ and the absolute values $\mathrm{F}$ and/or $\mathrm{M}$, depending on the movement type. An uncontrolled tipping can be assessed by $\mathrm{M}_{\mathrm{y}}$ and $\mathrm{M}_{\mathrm{y}} /\left(\mathrm{F}_{\mathrm{x}}+\mathrm{F}_{\mathrm{z}}\right) \cdot \mathrm{M}_{\mathrm{y}}$ defines the amount of tooth rotation along the desired direction, whereas $\mathrm{M}_{\mathrm{y}} /\left(\mathrm{F}_{\mathrm{x}}+\mathrm{F}_{\mathrm{z}}\right)$ provides information about the $\mathrm{C}_{\mathrm{ROT}}$ position. Higher $\mathrm{M}_{\mathrm{y}} /\left(\mathrm{F}_{\mathrm{x}}+\mathrm{F}_{\mathrm{z}}\right)$ values indicate a movement closer to the uncontrolled tipping.

Figure 11-a shows the full-field displacement map regarding the six frontal teeth for both the mandibular and the maxillary anatomies obtained by using the single divot configuration. Figures 11-b,f show the displacement maps for the maxillary (upper row) and mandibular (lower row) central incisors obtained for the five different aligner configurations, with the auxiliary elements located at the bracket height $(h=0)$.

Table 2 reports the analysed force system parameters for all the simulated scenarios. A preliminary analysis of the results shows that the orthodontic movement is better achieved by using auxiliary elements. In particular, the divots cause the highest displacement values both in single and double configurations.

The results reported in Table 2 have been aggregated in different plots in order to highlight the effectiveness of the various aligner layouts.

Figure 12 reports the numerical values of $\mathrm{M}_{\mathrm{y}}$ and $\mathrm{M}_{\mathrm{y}} /\left(\mathrm{F}_{\mathrm{x}}+\mathrm{F}_{\mathrm{z}}\right)$ of all the configurations when $h=0$. The same trends can be observed for both the mandibular and the maxillary incisors. For instance, the divot clearly increases the amount of $\mathrm{M}_{\mathrm{y}}$ with respect to the standard aligner. The attachment geometry brings to a significant improvement of the $\mathrm{M}_{\mathrm{y}} /\left(\mathrm{F}_{\mathrm{x}}+\mathrm{F}_{\mathrm{z}}\right)$ value. Moreover, the use of a double divot increases the moment $\mathrm{M}_{\mathrm{y}}$ with respect to the single divot configuration. Figure 13 evidences the effect of the vertical location of the single divot on the aligner effectiveness. The parameter $h$ does not significantly affect $\mathrm{M}_{\mathrm{y}} /\left(\mathrm{F}_{\mathrm{x}}+\mathrm{F}_{\mathrm{z}}\right)$, while an increment of $\mathrm{M}_{\mathrm{y}}$ can be observed for both maxillary and mandibular central incisors as $h$ increases. 
The numerical analysis evidences that the attachment effectiveness does not substantially change by varying its placement on the tooth crown; while a slight variation occurs by changing its orientation since a horizontal attachment brings to higher $\mathrm{M}_{\mathrm{y}}$ values with respect to a vertical attachment (Figure 14). Figure 15 reports the variations of the force system on the target teeth obtained by the double divot geometry and varying both height and mutual distance between the divots. Higher $\mathrm{M}_{\mathrm{y}}$ values can be observed when the $h$ values increase regardless the distance between the two divots.

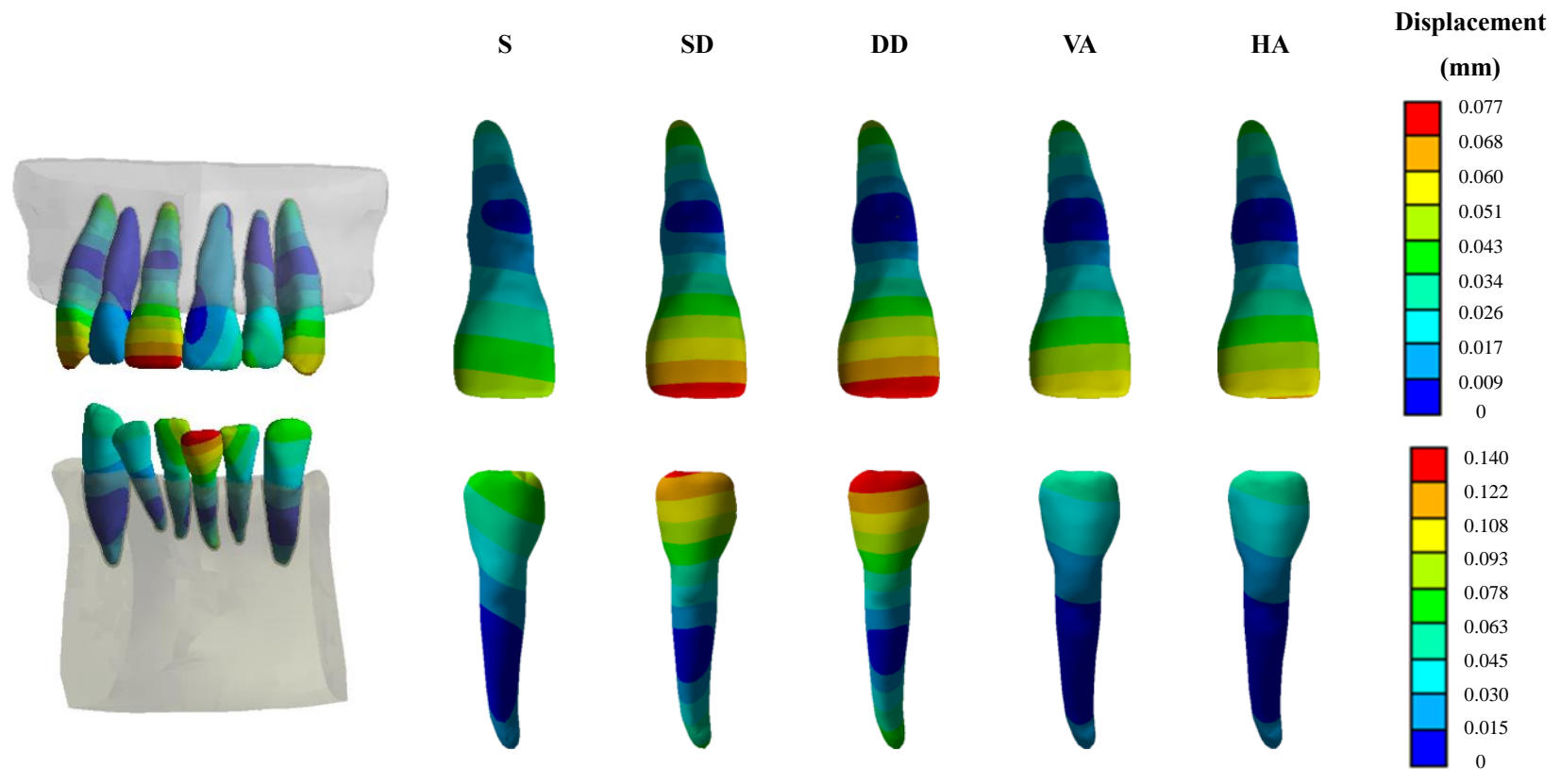

(a)

(b)

(c)

(d)

(e)

(f)

Figure 11 -Full-field displacement map regarding maxillary and mandibular dental arches obtained by using the single divot configuration (a), displacement maps for the maxillary (upper row) and mandibular (lower row) central incisors obtained for the five different aligner configurations: standard aligner (b), single divot aligner (c), double divot aligner (d), vertical attachment aligner (e), horizontal attachment aligner (f).
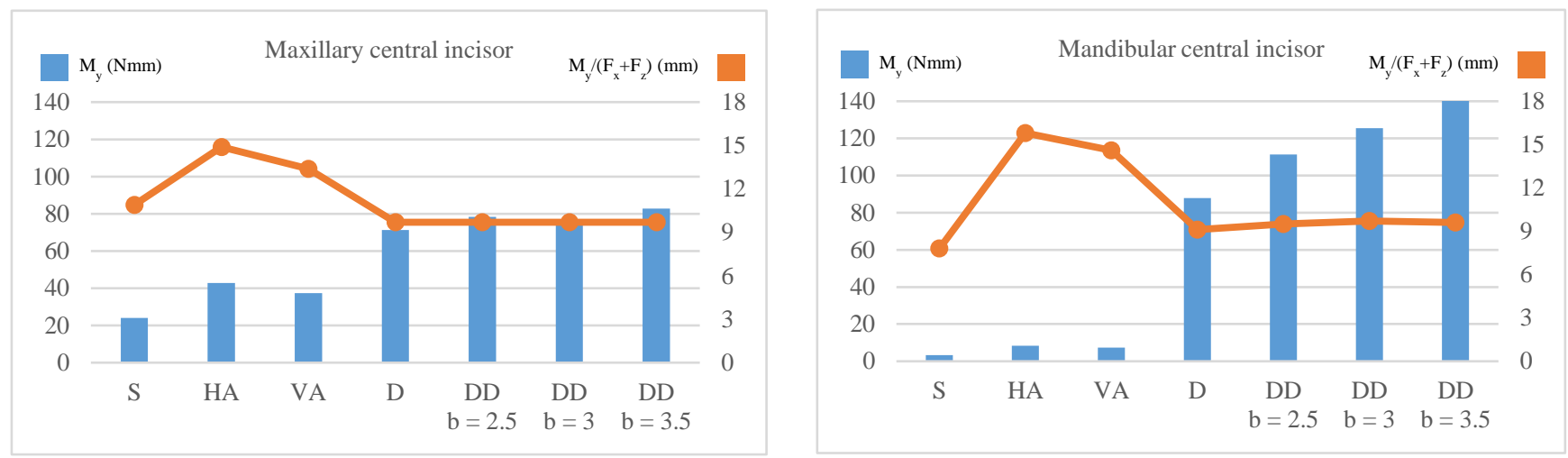

Figure 12 - Moment $\left(\mathrm{M}_{\mathrm{y}}\right)$ and Moment-to-force ratio $\left(\mathrm{M}_{\mathrm{y}} /\left(\mathrm{F}_{\mathrm{x}}+\mathrm{F}_{\mathrm{z}}\right)\right)$ for each aligner configuration with $h=0$. 
Single divot

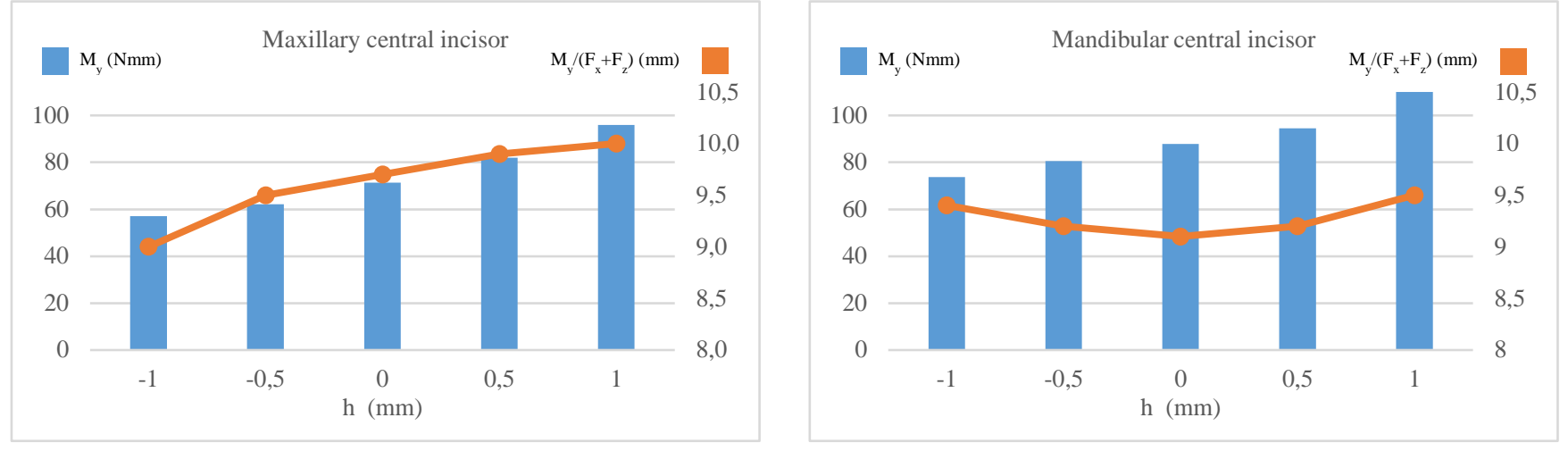

Figure 13 - Moment and Moment-to-force ratio for a single divot configuration with different $h$ values.

Horizontal attachment
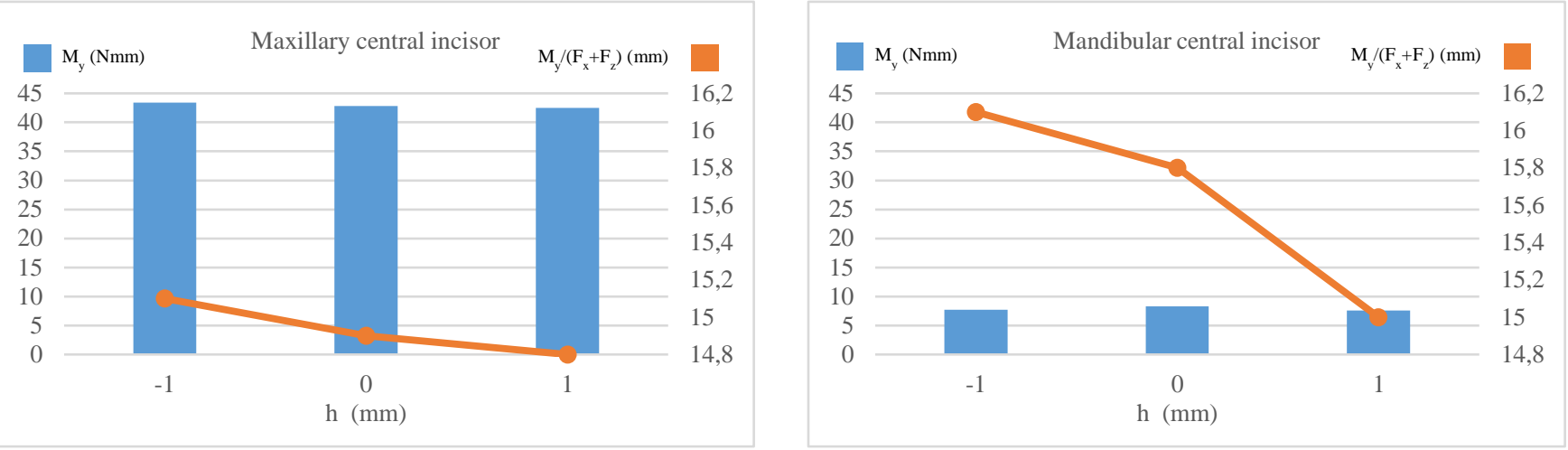

Vertical attachment
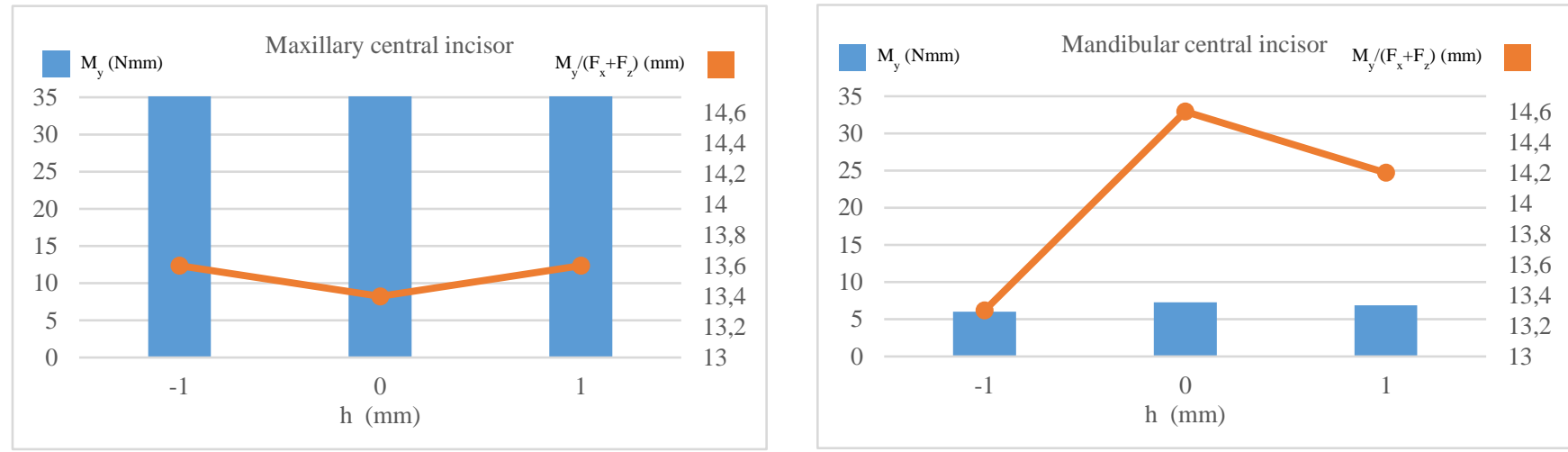

Figure 14 - Moment and Moment-to-force ratio for the horizontal and vertical configurations obtained by varying the height of the auxiliary elements. 
Double divot
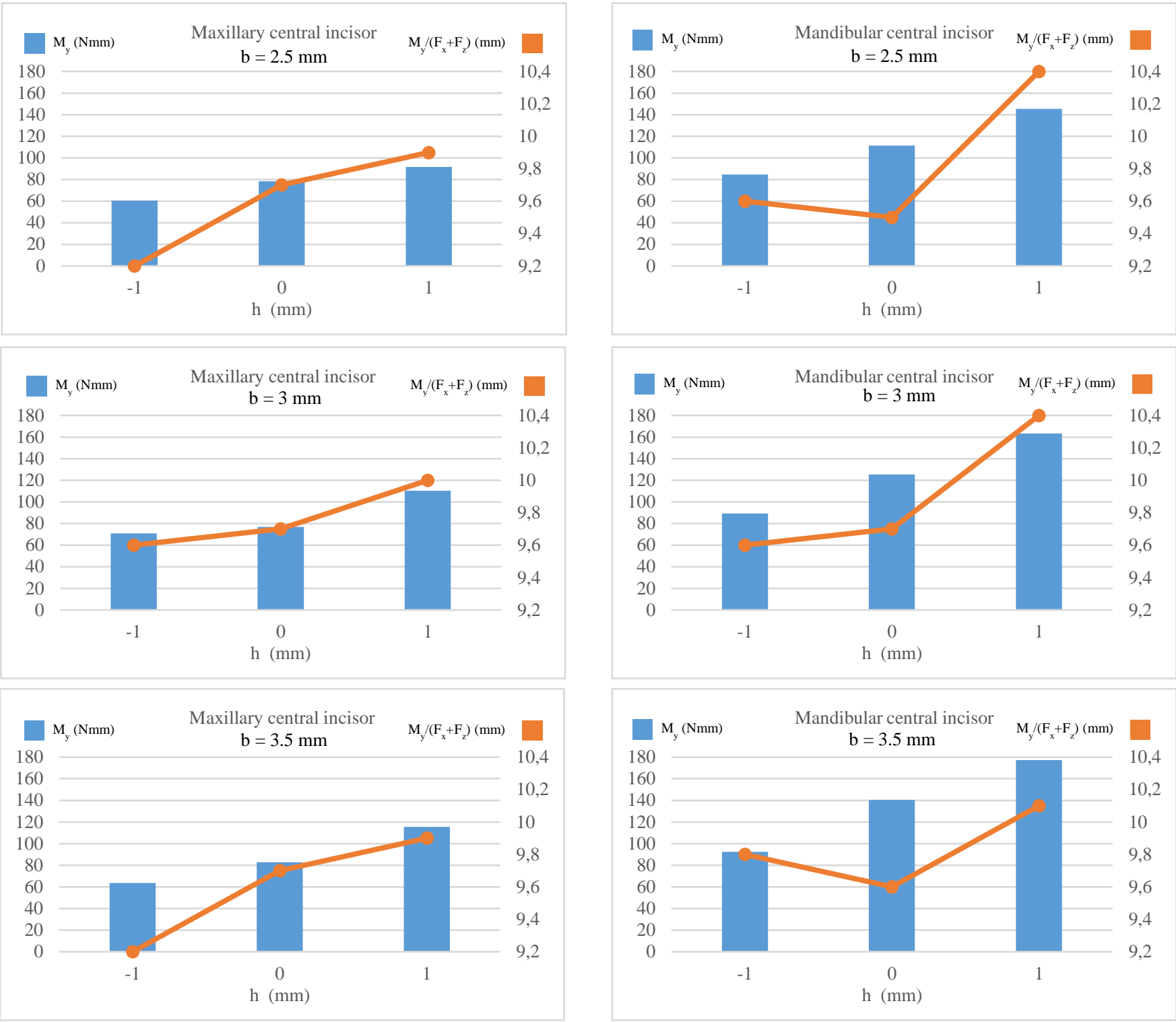

Figure 15 - Moment and Moment-to-force ratio for all the scenarios characterized by a double divot geometry.

\subsection{Discussion}

The results obtained by the finite element analyses have evidenced the effects owe to auxiliary elements in the treatment outcomes.

The 3D maps of Figure 11 show how the double divot configuration leads to the greatest tooth movement. The maxillary and mandibular incisor apexes incur in $0.077 \mathrm{~mm}$ and $0.14 \mathrm{~mm}$ displacements, respectively. The difference between the two displacement values can be ascribed to the different tooth dimensions. The divot geometry has the same size for both teeth, but the maxillary incisor volume $\left(627 \mathrm{~mm}^{3}\right)$ is almost three times greater than the mandibular incisor volume $\left(219 \mathrm{~mm}^{3}\right)$. Moreover, Table 2 and Figure 13 indicate that the single divot configuration generates a maximum $\mathrm{M}_{\mathrm{y}}=96 \mathrm{Nmm}$ and $\mathrm{M}_{\mathrm{y}}=110 \mathrm{Nmm}$ for the maxillary and mandibular incisors, respectively. The divot vertical displacement strongly affects the biomechanical effectiveness. For the maxillary incisor, $\mathrm{M}_{\mathrm{y}}$ increases from $57.1 \mathrm{Nmm}$ for $h=-1$ to $M_{y}=96 \mathrm{Nmm}$ for $h=1$. 
The same trend is obtained for the mandibular incisor with $\mathrm{M}_{\mathrm{y}}$ varying from $73.7 \mathrm{Nmm}$ to $110 \mathrm{Nmm}$. Hence, a vertical translation of $2 \mathrm{~mm}$ can enhance the resulting moment value by at least $50 \%$, since a greater distance between the divot location and $\mathrm{C}_{\mathrm{RES}}$ generates a higher moment at the $\mathrm{C}_{\mathrm{RES}}$ even without a variation in the force magnitude. The same tendency can be observed for the double divot geometry (Figure 15). The high sensitivity of the results to the vertical location of the divot suggests that during the design process the divot location should be carefully selected. A low magnitude of the force system could not activate the bone remodelling process. On the other hand, high values of the force system could cause root resorption. Hence, the divot should be placed in order to deliver the most appropriate loads according to the required tooth movement.

$\mathrm{M}_{\mathrm{y}}$ slightly increases by distancing the divots in the double configuration. The observed variations can be attributed to the non-symmetric crown shape, which introduces different force directions by distancing the two divots. This effect is a little bit more noticeable for the mandibular incisor than for the maxillary incisor. However, the $\mathrm{M}_{\mathrm{y}}$ increment is less significant with respect to the amount occurring by changing the divot vertical position. The use of a double divot geometry does not seem justifiable for an orthodontic tipping, since the load delivered by a single divot is already sufficient to obtain the required tooth movement [40].

The attachment geometry generates a higher $\mathrm{M}_{\mathrm{y}}$ onto the target teeth with respect to the standard configuration. The horizontal attachment provides a higher moment value compared to the vertical one. These results are in accordance with previous researches, which demonstrated the usefulness of attachments to enhance orthodontic treatments with aligners [13, 41]. However, these studies focused on the quantity of the delivered force system avoiding any analysis of the M:F parameter. Figure 12 shows that both vertical and horizontal attachments bring to more accurate movements in all the scenarios. This is confirmed by the $\mathrm{M}_{\mathrm{y}} /\left(\mathrm{F}_{\mathrm{x}}+\mathrm{F}_{\mathrm{z}}\right)$ values, which range from 13.3 to 16.1 among all the attachment-based scenarios. Meanwhile, the same parameter varies between 9 and 10.4 for the divot-based arrangements.

It is remarkable how $\mathrm{M}_{\mathrm{y}}$ values greatly vary between corresponding simulations of maxillary and mandibular incisors, nevertheless $\mathrm{M}_{\mathrm{y}} /\left(\mathrm{F}_{\mathrm{x}}+\mathrm{F}_{\mathrm{z}}\right)$ assumes similar values for the corresponding scenarios. This difference can be ascribed to the smaller height of the mandibular incisor with respect to the maxillary one. The loading condition has been introduced by a rotation of each target tooth around the $\mathrm{C}_{\mathrm{RES}}$ by $-0.3^{\circ}$. For this reason, the shorter tooth is subjected to a lower initial mismatch with respect to the aligner surface at the crown level.

The numerical simulations have showed a high dependency on the auxiliary element locations. This result should be used to improve the clinical practice. Usually, attachments are placed through accurate templates designed and manufactured by digital processes (CAD and RP). On the contrary, divot geometries are obtained by manually stamping the aligner with specific pliers on the basis of a visual guidance. This process is prone to errors, which can introduce uncertainties in the actual placement. Of course, an automatic procedure to physically create divot geometries onto the aligner surface would be highly desirable.

The proposed CDE methodology could be used to analyse various orthodontic conditions in order to retrieve the best design configuration for each clinical requirement. Multiple orthodontic movements for different 
teeth could also be analysed in order to identify a generalized rule to select the appropriate aligner's configuration.

Even if numerical simulations have been focused on the study of auxiliary elements, the developed pipeline could be used to study the influence of other aligner features (i.e., thickness and material properties). The material properties affect the clinical outcomes. Advisable material properties would be large spring-back, high stored energy, tolerance to mouth hostile environment, biocompatibility and low surface roughness. These peculiarities should be taken into high consideration when planning an aligner-based treatment and their effect could be studied by adopting the proposed approach. Moreover, the aligner thickness has a great influence on the magnitude of forces produced by the appliance $[3,42]$. In this paper, a uniform thickness has been considered to model the aligner shape, since the standard thermoforming process does not provide any control on the actual shell thickness. However, the CDE methodology might take into account non-uniform thicknesses, which could occur as a consequence of the thermoforming process.

The use of CBCT in clinical orthodontics is still a matter of broad concern due to the risk that unnecessary exposure to ionizing radiation might outweigh its benefits [43]. However, CBCT imaging is currently used in many clinical cases since it has demonstrated efficacy in altering or refining orthodontic treatment planning when teeth and root anomalies are present (e.g., impacted or unerupted teeth, transpositions, supernumeraries, root angulation, morphology and resorption) [44]. A further consideration is that CBCT has evolved at an impressive rate in the dental field since its introduction in 1998 [44]. Compared to the first systems, various hardware and software innovations have provided equipment with higher resolution and lower radiation doses, which could bring to a wider and safer use of CBCT in orthodontics in the near future [43]. It is also worth noting that in this paper, a multi-modality imaging approach has been used by integrating optical and tomographic scanning. However, technical literature has recently faced the problem of retrieving root anatomies by combining panoramic and/or lateral radiographs, without involving tomographic scanning [45-47]. This would bring to the reconstruction of complete tooth models, thus addressing questions regarding root movements, by exploiting minimally invasive imaging modalities. This research trend is justified by the fact that, in current orthodontic practice, both panoramic radiographs and lateral cephalograms are usually required for diagnostic purposes.

The presented framework, even if characterized by elaborate procedures (anatomical reconstruction, aligner's parametric modelling and finite element analyses) could be also used in a regular orthodontic procedure, at least for challenging clinical cases. An additional consideration lends further support to this position. Actually, the planning of aligner-based orthodontic treatments makes an extensive use of CAD tools. These computational tools could be enriched by introducing also modelling and simulation tools, thus providing a comprehensive knowledge of the whole scenario. 
The aim of this paper is at proposing a computational design and engineering framework to optimise the aligner attributes on the basis of patient-specific requirements.

The methodology is based on the digital reconstruction of anatomical models composed of teeth (complete of crowns and roots), periodontal ligaments, and bone tissue. The anatomical models are integrated with aligner models whose shape properly fits patient's dentition. Numerical analyses are performed to evaluate the behaviour of the appliance with the aim at guiding the clinical practice. The integration of several digital technologies (from medical imaging to numerical simulation) has demonstrated to be particularly effective in supporting the planning activity when auxiliary elements are introduced. Finite element analyses proved to be a powerful tool to study different aligner's configurations evidencing how auxiliary elements features might affect the loading system delivered by the aligner. For instance, the results of the test cases have pointed out that the loads elicited by the divot geometry, both in the single and double configuration, are higher than those provided by using attachments. Moreover, the numerical simulations have given a direct correlation between auxiliary element configurations and amounts of force/moment systems. The numerical results obtained in the presented work are in accordance to those obtained in clinical studies [48], which have compared the amount of predicted tooth movements with that achieved after treatment. In this context, future activity will regard the validation of numerical results with real clinical cases regarding patients, provided with CBCT scans, and undergoing to orthodontic corrections by polymeric aligners.

\section{References}

[1] Boyd RL. Esthetic orthodontic treatment using the invisalign appliance for moderate to complex malocclusions. J Dent Educ. 2008;72(8):948-67.

[2] Kohda N, Iijima M, Muguruma T, Brantley WA, Ahluwalia KS, Mizoguchi I. Effects of mechanical properties of thermoplastic materials on the initial force of thermoplastic appliances. Angle Orthod. 2013;83(3):476-83. doi:10.2319/052512-432.1.

[3] Hahn W, Dathe H, Fialka-Fricke J, Fricke-Zech S, Zapf A, Kubein-Meesenburg D, et al. Influence of thermoplastic appliance thickness on the magnitude of force delivered to a maxillary central incisor during tipping. Am J Orthod Dentofac Orthop. 2009;136(1):12.e1-7. doi:10.1016/j.ajodo.2008.12.015.

[4] Kravitz ND, Kusnoto B, Agran B, Viana G. Influence of attachments and interproximal reduction on the accuracy of canine rotation with invisalign - A prospective clinical study. Angle Orthod. 2008;78(4):682-7. doi:Doi 10.2319/060107-263.

[5] Martorelli M, Gerbino S, Giudice M, Ausiello P. A comparison between customized clear and removable orthodontic appliances manufactured using RP and CNC techniques. Dent Mater. 2013;29(2):E1-E10. doi:10.1016/j.dental.2012.10.011.

[6] Beers AC, Choi W, Pavlovskaia E. Computer-assisted treatment planning and analysis. Orthod Craniofac Res. 2003;6(Suppl. 1):117-25.

[7] Cuperus AMR, Harms MC, Rangel FA, Bronkhorst EM, Schols JGJH, Breuning KH. Dental models made with an intraoral scanner: A validation study. Am J Orthod Dentofac Orthop. 2012;142(3):308-13. doi:10.1016/j.ajodo.2012.03.031.

[8] Rossini G, Parrini S, Castroflorio T, Deregibus A, Debernardi CL. Efficacy of clear aligners in controlling orthodontic tooth movement: A systematic review. Angle Orthod. 2015;85(5):881-9. doi:10.2319/061614436.1 .

[9] Mencattelli M, Donati E, Cultrone M, Stefanini C. Novel universal system for 3-dimensional orthodontic force-moment measurements and its clinical use. Am J Orthod Dentofac Orthop. 2015;148(1):174-83. doi:10.1016/j.ajodo.2015.01.028. 
[10] Barbagallo LJ, Shen G, Jones AS, Swain MV, Petocz P, Darendeliler MA. A novel pressure film approach for determining the force imparted by clear removable thermoplastic appliances. Ann Biomed Eng. 2008;36(2):335-41. doi:10.1007/s10439-007-9424-5.

[11] Hahn W, Engelke B, Jung K, Dathe H, Fialka-Fricke J, Kubein-Meesenburg D, et al. Initial Forces and Moments Delivered by Removable Thermoplastic Appliances during Rotation of an Upper Central Incisor. Angle Orthod. 2010;80(2):239-46. doi:10.2319/033009-181.1.

[12] Elkholy F, Panchaphongsaphak T, Kilic F, Schmidt F, Lapatki BG. Forces and moments delivered by PET-G aligners to an upper central incisor for labial and palatal translation. $J$ Orofac Orthop. 2015;76(6):460-75. doi:10.1007/s00056-015-0307-3.

[13] Simon M, Keilig L, Schwarze J, Jung BA, Bourauel C. Forces and moments generated by removable thermoplastic aligners: Incisor torque, premolar derotation, and molar distalization. Am J Orthod Dentofac Orthop. 2014;145(6):728-36. doi:10.1016/j.ajodo.2014.03.015.

[14] Xia ZY, Chen J. Biomechanical validation of an artificial tooth-periodontal ligament-bone complex for in vitro orthodontic load measurement. Angle Orthod. 2013;83(3):410-7. doi:10.2319/041712-317.1.

[15] Cattaneo PM, Dalstra M, Melsen B. The finite element method: a tool to study orthodontic tooth movement. J Dent Res. 2005;84(5):428-33.

[16] Field C, Ichim I, Swain MV, Chan E, Darendeliler MA, Li W, et al. Mechanical responses to orthodontic loading: a 3-dimensional finite element multi-tooth model. American journal of orthodontics and dentofacial orthopedics : official publication of the American Association of Orthodontists, its constituent societies, and the American Board of Orthodontics. 2009;135(2):174-81. doi:10.1016/j.ajodo.2007.03.032.

[17] Qian HH, Chen J, Katona TR. The influence of PDL principal fibers in a 3-dimensional analysis of orthodontic tooth movement. Am J Orthod Dentofac Orthop. 2001;120(3):272-9. doi:10.1067/mod.2001.116085.

[18] Kojima Y, Fukui H. A numerical simulation of tooth movement by wire bending. Am J Orthod Dentofac Orthop. 2006;130(4):452-9. doi:10.1016/j.ajodo.2005.01.028.

[19] Canales C, Larson M, Grauer D, Sheats R, Stevens C, Ko CC. A novel biomechanical model assessing continuous orthodontic archwire activation. Am J Orthod Dentofac Orthop. 2013;143(2):281-90. doi:10.1016/j.ajodo.2012.06.019.

[20] Gomez JP, Pena FM, Martinez V, Giraldo DC, Cardona CI. Initial force systems during bodily tooth movement with plastic aligners and composite attachments: A three-dimensional finite element analysis. Angle Orthod. 2015;85(3):454-60. doi:10.2319/050714-330.1.

[21] Cai YQ, Yang XX, He BW, Yao J. Finite element method analysis of the periodontal ligament in mandibular canine movement with transparent tooth correction treatment. Bmc Oral Health. 2015;15(106). doi:10.1186/s12903-015-0091-x.

[22] Barone S, Paoli A, Razionale AV, Savignano R. Computer aided modelling to simulate the biomechanical behaviour of customised orthodontic removable appliances. Int J Interact Des Manuf. 2014. doi:10.1007/s12008-014-0246-z.

[23] Turner CH, Pavalko FM. Mechanotransduction and functional response of the skeleton to physical stress: The mechanisms and mechanics of bone adaptation. Journal of Orthopaedic Science. 1998;3(6):34655. doi:10.1007/s007760050064.

[24] Smith RJ, Burstone CJ. Mechanics of Tooth Movement. Am J Orthod Dentofac Orthop. 1984;85(4):294-307. doi:10.1016/0002-9416(84)90187-8.

[25] Viecilli RF, Budiman A, Burstone CJ. Axes of resistance for tooth movement: Does the center of resistance exist in 3-dimensional space? Am J Orthod Dentofac Orthop. 2013;143(2):163-72. doi:10.1016/j.ajodo.2012.09.010.

[26] Burstone CJ, Pryputniewicz RJ. Holographic Determination of Centers of Rotation Produced by Orthodontic Forces. Am J Orthod Dentofac Orthop. 1980;77(4):396-409. doi:10.1016/0002-9416(80)901050.

[27] Savignano R, Viecilli R, Paoli A, Razionale AV, Barone S. Nonlinear Dependency of Tooth Movement on Force System Directions. Am J Orthod Dentofac Orthop. 2016;149(6):8. doi:10.1016/j.ajodo.2015.11.025.

[28] Natali AN. Dental Biomechanics. London: Taylor \& Francis Ltd; 2003.

[29] Barone S, Paoli A, Razionale A. Creation of 3D Multi-Body Orthodontic Models by Using Independent Imaging Sensors. Sensors. 2013;13(2):2033-50. doi:10.3390/s130202033. 
[30] Barone S, Paoli A, Razionale AV. CT segmentation of dental shapes by anatomy-driven reformation imaging and B-spline modelling. International Journal for Numerical Methods in Biomedical Engineering. 2016;32(6):1-17. doi:10.1002/cnm.2747.

[31] Kazhdan M, Bolitho M, Hoppe H. Poisson surface reconstruction. In Proceedings of the fourth Eurographics symposium on Geometry processing. Cagliari, Sardinia, Italy: Eurographics Association; 2006. p. 61-70.

[32] Di Angelo L, Di Stefano P, Bernardi S, Continenza MA. A new computational method for automatic dental measurement: The case of maxillary central incisor. Comput Biol Med. 2016;70:202-9. doi:10.1016/j.compbiomed.2016.01.018.

[33] Dorow C, Schneider J, Sander FG. Finite Element Simulation of in Vivo Tooth Mobility in Comparison with Experimental Results. J Mech Med Biol. 2003;3(1):79-94. doi:10.1142/S0219519403000661.

[34] Ryokawa H, Miyazaki Y, Fujishima A, Miyazaki T, Maki K. The mechanical properties of dental thermoplastic materials in a simulated intraoral environment. Orthodontic Waves. 2006;65(2):64-72. doi:10.1016/j.odw.2006.03.003.

[35] Vollmer D, Bourauel C, Maier K, Jager A. Determination of the centre of resistance in an upper human canine and idealized tooth model. Eur J Orthodont. 1999;21(6):633-48. doi:10.1093/Ejo/21.6.633.

[36] Barone S, Paoli A, Neri P, Razionale AV, Giannese M. Mechanical and Geometrical Properties Assessment of Thermoplastic Materials for Biomedical Application. In: Eynard B, Nigrelli V, Oliveri MS, Peris-Fajarnes G, Rizzuti S, editors. Advances on Mechanics, Design Engineering and Manufacturing : Proceedings of the International Joint Conference on Mechanics, Design Engineering \& Advanced Manufacturing (JCM 2016), 14-16 September, 2016, Catania, Italy. Cham: Springer International Publishing; 2017. p. 437-46. doi: 10.1007/978-3-319-45781-9_44.

[37] Fill TS, Toogood RW, Major PW, Carey JP. Analytically determined mechanical properties of, and models for the periodontal ligament: Critical review of literature. $J$ Biomech. 2012;45(1):9-16. doi:10.1016/j.jbiomech.2011.09.020.

[38] Poppe M, Bourauel C, Jäger A. Determination of the Elasticity Parameters of the Human Periodontal Ligament and the Location of the Center of Resistance of Single-rooted Teeth. Journal of Orofacial Orthopedics. 2002;63(5):358-70. doi:10.1007/s00056-002-0067-8.

[39] Su MZ, Chang HH, Chiang YC, Cheng JH, Fuh LJ, Wang CY, et al. Modeling viscoelastic behavior of periodontal ligament with nonlinear finite element analysis. $J$ Dent Sci. 2013;8(2):121-8. doi:10.1016/j.jds.2013.01.001.

[40] Penedo ND, Elias CN, Pacheco MCT, Gouvêa JPd. 3D simulation of orthodontic tooth movement. Dental Press Journal of Orthodontics. 2010;15:98-108.

[41] Simon M, Keilig L, Schwarze J, Jung BA, Bourauel C. Treatment outcome and efficacy of an aligner technique--regarding incisor torque, premolar derotation and molar distalization. BMC oral health. 2014;14:68. doi:10.1186/1472-6831-14-68.

[42] Kwon JS, Lee YK, Lim BS, Lim YK. Force delivery properties of thermoplastic orthodontic materials. Am J Orthod Dentofac Orthop. 2008;133(2):228-34. doi:10.1016/j.ajodo.2006.03.034.

[43] American Academy of O, Maxillofacial R. Clinical recommendations regarding use of cone beam computed tomography in orthodontics. Position statement by the American Academy of Oral and Maxillofacial Radiology. Oral Surgery, Oral Medicine, Oral Pathology and Oral Radiology. 2013;116(2):238-57. doi:10.1016/j.oooo.2013.06.002.

[44] Kapila SD, Nervina JM. CBCT in orthodontics: assessment of treatment outcomes and indications for its use. Dentomaxillofac Rad. 2015;44(1). doi:10.1259/dmfr.20140282.

[45] Barone S, Paoli A, Razionale AV. Geometrical modeling of complete dental shapes by using panoramic X-ray, digital mouth data and anatomical templates. Comput Med Imag Grap. 2015;43:112-21. doi:10.1016/j.compmedimag.2015.01.005.

[46] Scheiner S, Hellmich C, Muller C, Bonitz L, Kober C. X-ray physics- and bone composition-based estimation of thickness characteristics from clinical mandibular radiographs. Comput Med Imag Grap. 2015;45:36-46. doi:10.1016/j.compmedimag.2015.06.005.

[47] Pei YR, Shi FH, Chen H, Wei J, Zha HB, Jiang RP, et al. Personalized Tooth Shape Estimation From Radiograph and Cast. Ieee T Bio-Med Eng. 2012;59(9):2400-11. doi:Doi 10.1109/Tbme.2011.2174993.

[48] Kravitz ND, Kusnoto B, BeGole E, Obrez A, Agran B. How well does Invisalign work? A prospective clinical study evaluating the efficacy of tooth movement with Invisalign. Am J Orthod Dentofac Orthop. 2009;135(1):27-35. doi:10.1016/j.ajodo.2007.05.018. 
Table 1 - Material properties used for the numerical simulations.

\begin{tabular}{|c|c|c|}
\hline & E $(\mathrm{MPa})$ & Poisson's ratio \\
\hline Tooth & 20000 & 0.3 \\
\hline Bone & 13800 & 0.3 \\
\hline Aligner & 1400 & 0.3 \\
\hline Attachment & 20000 & 0.3 \\
\hline
\end{tabular}


Table 2 - Force system parameters measured at the $\mathrm{C}_{\text {RES }}$.

\begin{tabular}{|c|c|c|c|c|c|c|}
\hline & \multirow[b]{2}{*}{$\boldsymbol{b}(\mathrm{mm})$} & \multirow[b]{2}{*}{$\boldsymbol{h}(\mathrm{mm}$} & \multicolumn{2}{|c|}{$\begin{array}{c}\text { Maxillary central } \\
\text { incisor }\end{array}$} & \multicolumn{2}{|c|}{$\begin{array}{c}\text { Mandibular central } \\
\text { incisor }\end{array}$} \\
\hline & & & $\boldsymbol{M}_{\boldsymbol{y}}(\mathrm{Nmm})$ & $\begin{array}{c}\boldsymbol{M}_{\boldsymbol{y}} /\left(\boldsymbol{F}_{\boldsymbol{x}}+\boldsymbol{F}_{z}\right) \\
(\mathrm{mm})\end{array}$ & $\boldsymbol{M}_{\boldsymbol{y}}(\mathrm{Nmm})$ & $\begin{array}{c}\boldsymbol{M}_{\boldsymbol{y}} /\left(\boldsymbol{F}_{\boldsymbol{x}}+\boldsymbol{F}_{z}\right) \\
(\mathrm{mm})\end{array}$ \\
\hline HA & - & -1 & 43.4 & 15.1 & 7.7 & 16.1 \\
\hline HA & - & 0 & 42.8 & 14.9 & 8.3 & 15.8 \\
\hline HA & - & 1 & 42.5 & 14.8 & 7.6 & 15.0 \\
\hline VA & - & -1 & 38 & 13.6 & 6.0 & 13.3 \\
\hline VA & - & 0 & 37.4 & 13.4 & 7.3 & 14.6 \\
\hline VA & - & 1 & 37.3 & 13.6 & 6.9 & 14.2 \\
\hline SD & - & -1 & 57.1 & 9.0 & 73.7 & 9.4 \\
\hline SD & - & -0.5 & 62.1 & 9.5 & 80.6 & 9.2 \\
\hline SD & - & 0 & 71.3 & 9.7 & 87.8 & 9.1 \\
\hline SD & - & 0.5 & 82 & 9.9 & 94.5 & 9.2 \\
\hline SD & - & 1 & 96 & 10.0 & 110.0 & 9.5 \\
\hline DD & 2.5 & -1 & 60.5 & 9.2 & 84.6 & 9.6 \\
\hline DD & 3 & -1 & 70.8 & 9.6 & 89.2 & 9.6 \\
\hline DD & 3.5 & -1 & 63.6 & 9.2 & 92.4 & 9.8 \\
\hline DD & 2.5 & 0 & 78.3 & 9.7 & 111.4 & 9.5 \\
\hline DD & 3 & 0 & 76.9 & 9.7 & 125.4 & 9.7 \\
\hline DD & 3.5 & 0 & 82.8 & 9.7 & 140.5 & 9.6 \\
\hline DD & 2.5 & 1 & 91.7 & 9.9 & 145.4 & 10.4 \\
\hline DD & 3 & 1 & 110.3 & 10.0 & 163.4 & 10.4 \\
\hline DD & 3.5 & 1 & 115.6 & 9.9 & 177.2 & 10.1 \\
\hline $\mathbf{S}$ & - & - & 24 & 10.9 & 3.4 & 7.8 \\
\hline
\end{tabular}

